\title{
Aprendizagem induzida pela instrução em contexto de organizações e trabalho: uma análise crítica da literatura
}

\author{
Thaís Zerbini ${ }^{\mathrm{I}}$ e Gardênia Abbad ${ }^{\mathrm{II}}$ \\ ${ }^{\text {I }}$ Faculdade de Filosofia, Ciências e Letras de Ribeirão Preto, Universidade de São Paulo \\ ${ }^{\text {II }}$ Universidade de Brasília
}

\begin{abstract}
O atendimento às novas exigências do mercado de trabalho, caracterizado por transformações sociais e tecnológicas, exige que as organizações que compõem o sistema produtivo desenvolvam estratégias de atualização contínua de suas competências organizacionais e individuais. Além disso, é fato que um dos grandes desafios do Brasil é promover o desenvolvimento sustentável econômico e social em diversas regiões. Programas de treinamento, desenvolvimento e educação de pessoal (TD\&E) nas empresas e de formação e qualificação profissional em ambiente aberto contribuem para que esse desenvolvimento ocorra. Torna-se necessário entender, portanto, como ocorrem os processos de aprendizagem e, principalmente, de transferência de aprendizagem para diferentes ambientes corporativos e profissionais. O presente artigo pretende contribuir com a área de psicologia organizacional e do trabalho, ao apresentar uma análise crítica da literatura sobre o processo de aprendizagem induzida pela instrução em contexto de organizações e trabalho. Para tanto, são discutidos os conceitos, as teorias e as abordagens relacionadas ao processo de aprendizagem humana no trabalho, com ênfase na aprendizagem induzida pela instrução. Analisam-se também os conceitos e as características dos processos formais que promovem a aprendizagem induzida no trabalho: ações de treinamento, desenvolvimento e educação de pessoal, formação e qualificação profissional.
\end{abstract}

Palavras-chave: Aprendizagem humana, Aprendizagem induzida, Treinamento, Desenvolvimento, Educação.

Learning induced by instruction in context of organizations and work: a critical literature analysis

The answer to the new demands of the labor market, characterized by social and technological changes, requires the development of strategies from the production system organizations for continuous updating of their individual and organizational skills. Moreover, it is a fact that one of the great challenges of Brazil is promoting a sustainable economic and social development in various regions. Programs of training, development and education in the organizations and professional qualification in an open environment contribute to this development. It is necessary to understand how the learning processes and the transfer of learning to different environments and corporate professionals occurs. This article aims to contribute to the field of organizational and work psychology to make a critical analysis of literature about the learning process induced by instruction in the context of organizations and work. To achieve this goal, we discuss the concepts, theories and approaches related to the human learning process at work with emphasis on learning induced by instruction. We also examine the concepts and characteristics of formal procedures that promote learning induced at work: programs of training, development and education of personnel, training and professional qualification.

Keywords: Human learning, Learning induced, Training, Development, Education.

\section{Introdução}

discussão sobre educação e qualificação para o trabalho e suas relações com o
desenvolvimento do país tem ocorrido com muita frequência nas pautas de reuniões de
trabalhadores, empresários, governos, pesquisadores e profissionais liberais. O atendimento às
novas exigências do mercado de trabalho, caracterizado por transformações sociais e
tecnológicas, exige que empresas, escolas técnicas, órgãos de governo, entre outras instituições
que compõem o sistema produtivo, desenvolvam estratégias de atualização contínua de suas
competências organizacionais e individuais. Além disso, é fato que um dos grandes desafios 
brasileiros é promover o desenvolvimento sustentável econômico e social em diversas regiões. Programas de treinamento, desenvolvimento e educação de pessoal (TD\&E) nas empresas e de formação e qualificação profissional em ambiente aberto contribuem para que esse desenvolvimento ocorra.

Torna-se necessário entender, portanto, como ocorrem os processos de aprendizagem e, principalmente, os de transferência de aprendizagem para diferentes ambientes corporativos e profissionais. Para possibilitar o estudo desses fenômenos, pesquisadores e profissionais interessados em identificar formas de garantir a melhoria do desempenho no trabalho, procuram investigar o processo de aquisição de conhecimentos, habilidades e atitudes (CHAs) por meio de ações de TD\&E, formação e qualificação profissional. Tais eventos são considerados ações formais que induzem a aprendizagem em organizações e em diversos ambientes de trabalho.

Em função do aumento da demanda por ações desse tipo, empresas e organizações passaram a desenvolver e a oferecer programas de educação corporativa, formação e qualificação profissional mediante a utilização de novas tecnologias de comunicação e informação, que possibilitam um alcance maior de pessoas do que cursos presenciais. Nesse sentido, o presente artigo pretende contribuir com a área de psicologia organizacional e do trabalho, ao apresentar uma análise crítica da literatura sobre o processo de aprendizagem induzida pela instrução em contexto de organizações e trabalho.

São discutidos, inicialmente, os conceitos, as teorias e as abordagens relacionados ao processo de aprendizagem humana no trabalho, com ênfase na aprendizagem induzida pela instrução. Em seguida, discutem-se os conceitos e as características dos processos formais que promovem a aprendizagem induzida no trabalho: ações de TD\&E, presenciais e a distância, formação e qualificação profissional. Ao final do artigo, apresentam-se as considerações finais sobre o tema.

\section{Aprendizagem humana no trabalho}

Segundo Abbad, Nogueira e Walter (2006), um dos principais objetivos das pesquisas em TD\&E consiste em identificar as condições necessárias para o indivíduo adquirir determinados conhecimentos, habilidades e atitudes (CHAs) exigidos pelo trabalho. As organizações atuais, preocupadas com a manutenção da competitividade no mercado, mostramse interessadas em entender como ocorrem os processos de aprendizagem e, principalmente, os processos de transferência para os diferentes níveis da organização. Para tanto, as pesquisas de TD\&E apoiam-se em abordagens teóricas que descrevem o processo de aprendizagem individual e que investigam a influência de variáveis individuais e ambientais sobre a aprendizagem.

Antes de iniciar a discussão sobre o conceito e as abordagens teóricas de aprendizagem, é importante diferenciar, no contexto de organizações e trabalho, a aprendizagem natural ou espontânea e a aprendizagem induzida. Segundo Abbad e Borges-Andrade (2004), a aprendizagem natural não necessita de procedimentos sistemáticos para ocorrer. Ao contrário, esse processo pode ocorrer por observação, conversas informais ou formais em reuniões e encontros, consulta a fontes de informação diversas, entre outros. Já a aprendizagem induzida ocorre mediante planejamento sistemático de procedimentos e estratégias instrucionais visando ao alcance de determinados CHAs, também previamente delineados. Segundo Abbad et al. (2006), essas situações de aprendizagem são conhecidas como as ações formais de TD\&E em contexto corporativo, formação e qualificação profissional em ambientes de trabalho diversos. 
Abbad et al. (2006) ressaltam a importância de entender as teorias de aprendizagem em pesquisas sobre treinamento e comportamento em organizações e trabalho. $O$ processo de atualização contínua das competências individuais e organizacionais, em um ambiente que muda constantemente suas exigências técnicas para se adequar ao mercado competitivo, tornase extremamente complexo. Por isso, os autores ressaltam a importância do estudo de teorias e resultados de pesquisas para fundamentar a prática profissional e a aprendizagem contínua no trabalho.

Segundo Pantoja e Borges-Andrade (2002), o termo aprendizagem possui uma grande variedade de definições, dependendo da teoria adotada pelo autor. De forma geral, os estudiosos da área caracterizam aprendizagem como um processo de mudanças que ocorre no indivíduo e que não é resultante da maturação ou de outro conceito que está associado à idade ou fases da vida. Abbad e Borges-Andrade (2004) definem aprendizagem como um processo psicológico, que ocorre no nível do indivíduo, essencial para a sobrevivência dos seres humanos no decorrer de todo o seu desenvolvimento, principalmente no mercado de trabalho. Portanto, as expressões aprendizagem organizacional e organizações que aprendem devem ser interpretadas metaforicamente. Tais expressões referem-se às organizações que promovem o compartilhamento de aprendizagens individuais e grupais, sendo possível, dessa maneira, a ocorrência da transferência de aprendizagem do nível individual para os outros níveis da organização.

Para Gagné (1985), o indivíduo demonstra que aprendeu algo novo ao executar, após um determinado evento instrucional, os comportamentos descritos nos objetivos instrucionais inicialmente propostos. Similarmente a essa definição, Pantoja e Borges-Andrade (2002) definem aprendizagem como o processo no qual o indivíduo, em interação com o ambiente, adquire e retém um novo conhecimento, habilidade ou atitude e que, posteriormente, pode ser observada em uma mudança de comportamento. Pilati e Abbad (2005) discutem os componentes do conceito aprendizagem (aquisição, retenção, generalização) relacionados aos conceitos de transferência de aprendizagem e impacto do treinamento no trabalho. A relação entre os conceitos foi utilizada pelos autores para descrever o processo de mudança comportamental como resultado de ações instrucionais em contexto organizacional. A Figura 1 apresenta o esquema gráfico dos conceitos relacionados.

Figura 1: Esquema gráfico dos conceitos relacionados à aprendizagem

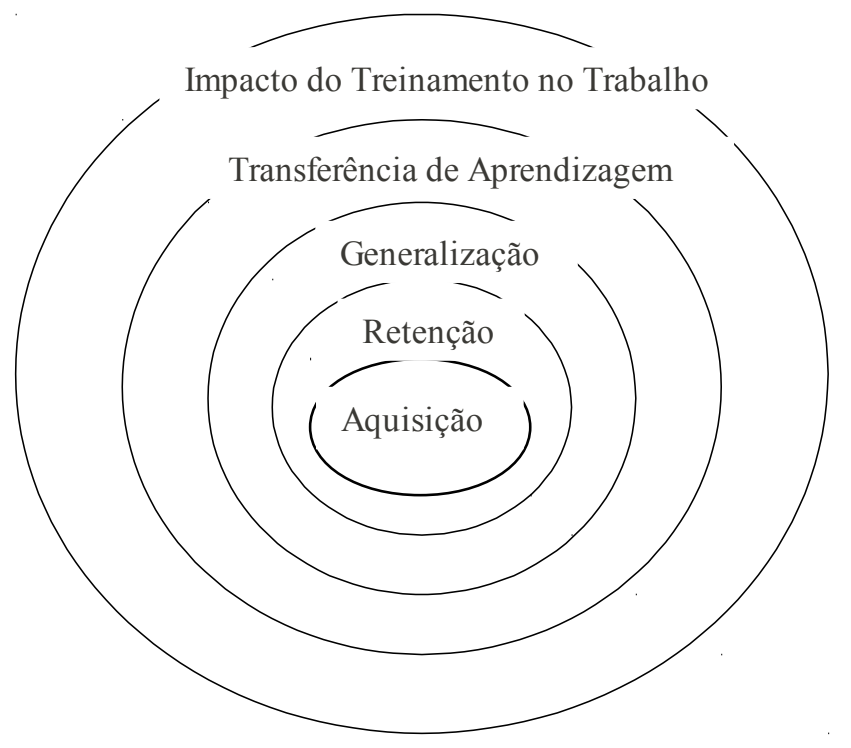

Fonte: Pilati e Abbad (2005). 
Aquisição, segundo os autores, define-se como o processo básico de apreensão de conhecimentos, habilidades e atitudes, desenvolvido ao longo da ação instrucional. Esse conceito se refere apenas a uma das etapas do processo de aprendizagem e depende de memorização para se concretizar. Retenção é o processo imediatamente posterior à aquisição e se refere ao armazenamento dos CHAs na memória de longo prazo. Para Abbad e BorgesAndrade (2004), retenção é a quantidade de tempo em que os CHAs adquiridos continuam a ser utilizados no ambiente de transferência após o término do programa instrucional. A generalização pode ser definida como o grau em que os CHAs adquiridos são utilizados no ambiente de transferência em contextos e condições diferentes daquelas que foram desenvolvidas durante eventos de TD\&E (Abbad \& Borges-Andrade, 2004; Ford \& Kraiger, 1995).

A transferência de aprendizagem pode ser definida como a aplicação efetiva no trabalho dos CHAs adquiridos em ação instrucional (Pilati \& Abbad, 2005). Abbad e Borges-Andrade (2004) definem transferência de aprendizagem como sendo as mudanças no indivíduo verificadas em atividades e situações diferentes daquelas em que ocorreu a aquisição, podendo variar em sentido (lateral e vertical) e direção (positiva e negativa). A transferência lateral se refere a um tipo de generalização que inclui desempenhos que não foram diretamente aprendidos, mas que são semelhantes aos aprendidos, ou seja, apresentam o mesmo grau de complexidade. A transferência vertical envolve aprendizagem de capacidades mais complexas a partir de capacidades mais simples. A transferência é positiva quando os $\mathrm{CHAs}$ adquiridos facilitam o desempenho do indivíduo no ambiente de transferência e negativa quando o prejudicam (Abbad, 1999). Segundo Abbad e Borges-Andrade (2004), o conceito de transferência inclui o de retenção e o de generalização. Os autores ressaltam que retenção e generalização são consideradas condições necessárias, porém não suficientes, ao uso eficaz no trabalho dos desempenhos aprendidos.

O conceito de transferência de aprendizagem, descrito anteriormente, foi denominado por Hamblin (1978) de impacto do treinamento em profundidade, para distinguir efeitos diretos e específicos de um evento instrucional de outros, mais gerais e não extraídos diretamente dos objetivos de ensino, por ele denominado impacto em amplitude. Segundo Pilati e Abbad (2005), a transferência de aprendizagem é o indicador mais utilizado em pesquisas de TD\&E para verificar a aplicação de CHAs no trabalho. Entretanto, os autores alertam que, caso o objetivo do profissional de treinamento seja medir os efeitos da ação instrucional no desempenho global, atitudes e motivação do indivíduo, a medida de transferência de aprendizagem (ou impacto do treinamento em profundidade) não é suficiente. $\mathrm{O}$ indivíduo que aplica os $\mathrm{CHAs} \mathrm{no} \mathrm{trabalho} \mathrm{não}$ apresentará, necessariamente, mudanças globais em seu comportamento.

Pilati e Abbad (2005) ressaltam que um dos grandes interesses das organizações é verificar o efeito do treinamento em longo prazo nos níveis de desempenho do indivíduo, bem como identificar as atitudes e as condições necessárias para que tais níveis melhorem e permaneçam em suas atividades diárias. Portanto, os autores concluem que tal efeito não pode ser descrito e medido como transferência de aprendizagem apenas, mas sim como impacto do treinamento no trabalho, definido como sendo a influência da ação instrucional no desempenho global, nas atitudes e na motivação subsequentes do participante de tal ação. Como citado anteriormente, esse fenômeno é nomeado por Hamblin (1978) de impacto em amplitude.

Outra expressão muito usada em psicologia organizacional é transferência de treinamento. Segundo análise conceitual realizada por Abbad (1999), os termos transferência de treinamento, transferência de aprendizagem e impacto no cargo são vistos como sinônimos pela área de psicologia organizacional. A autora ressalta ainda que transferência de treinamento e transferência de aprendizagem são expressões utilizadas em pesquisas que avaliam os resultados de ações de treinamento sobre o desempenho dos participantes em atividades similares às adquiridas ao longo do curso. 
Para os pesquisadores Baldwin e Ford (1988) e Ford e Kraiger (1995), o termo transferência de treinamento consiste na aplicação eficaz, no contexto de trabalho, dos CHAs ao longo da ação instrucional. Entretanto, Abbad (1999) ressalta que nem todas as situações que geram aprendizagem em organizações são ações formais de TD\&E. O processo de aprendizagem natural, como mencionado anteriormente, não necessita de procedimentos sistemáticos para ocorrer. Diante disso, a psicologia organizacional também busca investigar outras formas de transmissão de conhecimento e aprendizagem.

Segundo Abbad (1999), as expressões usadas com mais frequência em psicologia organizacional para avaliar os efeitos de atividades informais que promovem a transmissão de conhecimentos e tecnologias de trabalho são transferência de aprendizagem ou transferência de conhecimentos. Para a autora, nesses casos, o uso do conceito de transferência não contempla o efeito de eventos instrucionais planejados e formais sobre o desempenho subsequente do indivíduo no trabalho. A expressão transferência de aprendizagem, segundo a referida autora, tem origem na psicologia experimental, área na qual o termo adquire um uso mais genérico. Por outro lado, em estudos sobre avaliação de treinamento, por exemplo, o termo transferência de treinamento assume um uso mais específico, que se refere à aplicação eficaz, no ambiente de trabalho, dos CHAs adquiridos em situações de treinamento.

Outra característica importante presente na análise conceitual de transferência de treinamento realizada por Abbad (1999) é a natureza disposicional daquilo que é transferido do treinamento para o local de trabalho. Em psicologia organizacional, esse termo está associado ao fato dos treinamentos ensinarem novas habilidades, capacidades ou atitudes e novas formas de desempenhar antigas tarefas, visando à aplicação eficaz dos comportamentos aprendidos em outras situações ou tarefas no ambiente de trabalho. Para a autora, não interessam à psicologia organizacional apenas as medidas de retenção tomadas imediatamente após o treinamento, como ocorre na psicologia experimental. Em função disso, a natureza disposicional dos conceitos de habilidades, capacidades e atitudes precisa ser investigada em longo prazo no contexto de trabalho do indivíduo, pois o que mais interessa para os pesquisadores da área é avaliar a influência das ações instrucionais sobre os níveis de desempenho, bem como identificar fatores restritivos e facilitadores do uso daquelas habilidades, capacidades e atitudes e as condições necessárias para que tais níveis melhorem.

Diante do exposto, verifica-se que o conceito de transferência de aprendizagem, descrito por Pilati e Abbad (2005), é similar ao conceito de transferência de treinamento apresentado por Abbad (1999). Entretanto, em função da análise conceitual de Abbad (1999) apontar para o fato de que o conceito de transferência de aprendizagem também vem sendo utilizado para avaliar os efeitos de atividades informais em ambientes de trabalho e por ser um conceito com origem na psicologia experimental, em pesquisas sobre ações formais de TD\&E, formação e qualificação profissional, ainda assim, o conceito de transferência de treinamento é mais adequado, já que ele assume um uso mais específico ao se referir à aplicação eficaz, no ambiente de trabalho, dos CHAs adquiridos em situações formais de aprendizagem.

O conceito transferência de treinamento pode ser visto como sinônimo do conceito de impacto do treinamento em profundidade descrito por Hamblin (1978). No entanto, em eventos de formação e qualificação profissional, que geralmente ocorrem em ambientes abertos de trabalho, o termo mais adequado é o de transferência de treinamento, pois Hamblim (1978) restringe a utilização do termo impacto do treinamento em profundidade ao terceiro nível de avaliação proposto por ele, comportamento no cargo. Porém, em eventos de qualificação profissional, geralmente, os indivíduos têm interesse em adquirir novos conhecimentos e habilidades para trabalhos diversificados e não necessariamente mantêm vínculos empregatícios com a organização que oferta o curso. Portanto, não é possível observar os efeitos da ação instrucional no comportamento subsequente do indivíduo em seu cargo dentro da organização. $\mathrm{O}$ ambiente de transferência é diversificado nesse caso, podendo o indivíduo aplicar os conhecimentos adquiridos em qualquer trabalho. 
Definidos os conceito de aprendizagem, de transferência de aprendizagem, de transferência de treinamento e os outros termos correlatos, discutem-se a seguir algumas abordagens e teorias de aprendizagem que embasam o planejamento de ações formais de aprendizagem.

\section{Teorias e abordagens de aprendizagem}

Visando garantir que um maior número de funcionários transfira os conhecimentos, habilidades e atitudes para a o local de trabalho, as organizações investem grandes quantias em ações formais de TD\&E com a finalidade de desenvolver competências complexas que serão úteis para o crescimento da organização. Como discutido anteriormente, Abbad et al. (2006) ressaltam a importância de aplicar os achados provenientes de pesquisas sobre aprendizagem para aumentar a efetividade de ações de TD\&E, tão necessárias àqueles que necessitam de atualização contínua das competências profissionais. Antes de iniciar a discussão sobre teorias e abordagens de aprendizagem, é importante comentar, brevemente, as distinções entre os termos teorias de aprendizagem, teorias de desenho instrucional e teorias instrucionais, como destacado por Abbad et al. (2006).

De acordo com os autores, as teorias de aprendizagem apresentam classificação descritiva, pois trabalham com os aspectos subjacentes à ocorrência da aprendizagem, como por exemplo, teorias de processamento da informação. Tais teorias não ensinam o passo-a-passo de um planejamento instrucional, mas sim, as razões da utilização de determinadas prescrições. Entende-se que as teorias de desenho instrucional são específicas e descrevem detalhadamente os componentes e etapas para elaboração de planejamentos instrucionais, por isso, a sua classificação é prescritiva. As teorias instrucionais, segundo Abbad et al. (2006), em geral, são prescritivas, pois descrevem as condições externas mais adequadas à ocorrência da aprendizagem, mediante análise de determinadas condições internas do aprendiz. Portanto, teorias instrucionais também podem ser classificadas como descritivas, já que utilizam princípios básicos das teorias de aprendizagem que auxiliam nessas prescrições.

Abbad e Borges-Andrade (2004) destacam duas abordagens principais relacionadas às teorias de aprendizagem que contemplam as mudanças que ocorrem no indivíduo: a behaviorista e a cognitivista. Na tradição behaviorista (teorias S-R), o foco é a mudança de comportamento $(R)$, resultante da interação do indivíduo com seu ambiente $(S)$. A abordagem cognitivista (teorias S-O-R) enfatiza que a mudança de comportamento (R) provocada pela interação com o ambiente (S), resultaria, antes, em processos mentais ou na aquisição de conhecimentos, habilidades e atitudes $(\mathrm{O})$, que poderiam ser inferidos a partir daquelas mudanças.

Abbad et al. (2006) destacam que a abordagem construtivista (construção do conhecimento a partir de experiências do aprendiz e suas interações com o mundo) passou a ser utilizada em planejamentos de ações instrucionais por profissionais da área de organizações e trabalho. Outrora, tal abordagem era mais utilizada pela psicologia escolar e pela pedagogia. A abordagem mais utilizada pela psicologia instrucional nas últimas décadas, segundo os autores, é a cognitivista, de caráter objetivista. Com o crescimento de projetos corporativos em educação a distância, a abordagem cognitivista continua predominante, porém, apresentando também características construtivistas.

As teorias instrucionais cognitivistas compõem o referencial teórico predominante utilizado nas discussões dos processos de aprendizagem induzida realizadas no presente artigo. A escolha se justifica pelas análises feitas por Abbad (1999), as quais indicam que as teorias instrucionais, principalmente a de Gagné (1985) e a de Bloom, Engelhart, Furst, Hill, e 
Krathwohl (1972a, 1972b), ajudam na compreensão sobre como o aprendiz adquire competências, o que, por sua vez, possibilita o aperfeiçoamento de procedimentos e técnicas instrucionais.

Além disso, Abbad e Borges-Andrade (2004) alertam para a necessidade de utilizar princípios de aprendizagem e de tecnologias instrucionais no contexto de organizações e trabalho que facilitem a criação de condições adequadas à aprendizagem, retenção e transferência positiva dessas competências para o trabalho. Os autores enfatizam que um dos grandes desafios da área de treinamento é "garantir um alto grau de estruturação de eventos instrucionais e, ao mesmo tempo, respeitar as diferenças individuais" (p. 258). Segundo Abbad e Borges-Andrade (2004), as taxonomias de resultados ou objetivos educacionais são exemplos de conhecimentos técnicos que facilitam o planejamento, a execução e a avaliação de ações formais de TD\&E voltadas à aprendizagem em ambientes organizacionais diversos.

Bloom et al. (1972a, 1972b) elaboraram uma taxonomia para cada um dos domínios de aprendizagem existentes, quais sejam: o cognitivo, o afetivo e o psicomotor. Segundo Rodrigues Jr. (2006), taxonomia é um termo de origem grega que significa uma estrutura de sucessão fixa. Em uma taxonomia deve existir um sentido de hierarquia, apresentando sequência (eixo comum) e cumulatividade entre os níveis propostos. As taxonomias devem representar resultados de aprendizagem, sendo que, categorias de resultados de aprendizagem mais complexas dependem das mais simples para ocorrerem. Segundo o referido autor, cada taxonomia deve orientar-se por um princípio ordenador de categorias, ou seja, todas as categorias devem fazer parte de um mesmo continuum. No domínio cognitivo, o princípio organizador é a complexidade dos processos intelectuais; no afetivo, é a internalização de atitudes e valores; e no psicomotor, é a automatização de movimentos.

Abbad, Zerbini, Carvalho e Meneses (2006) ressaltam que os comportamentos resultantes dos domínios cognitivo, afetivo e psicomotor não ocorrem separadamente durante a aprendizagem dos indivíduos. A separação em três domínios é meramente didática e objetiva facilitar a definição de procedimentos instrucionais (meios e estratégias de ensino, métodos de avaliação etc.) de acordo com o comportamento predominante esperado do indivíduo após o evento instrucional.

Vale discutir, no entanto, mesmo que brevemente, a validade empírica das taxonomias de objetivos educacionais. Segundo Rodrigues Jr. (2006), alguns estudos analisados indicaram que o sentido de hierarquia e cumulatividade nem sempre foram mantidos, principalmente nas taxonomias dos domínios cognitivo e afetivo. Entretanto, segundo o autor, tais achados não impedem a utilização das taxonomias para planejar, executar e avaliar treinamentos, podendo o profissional de treinamento, eventualmente, ajustar a ordem hierárquica da taxonomia de acordo com o perfil do treinamento.

Gagné (1968a), um dos principais representantes em estudos sobre o processo de aprendizagem, muito contribuiu para o planejamento da aprendizagem e da instrução. $\mathrm{O}$ autor propôs o modelo de aprendizagem cumulativa, que consiste na contribuição da aprendizagem no desenvolvimento intelectual do ser humano visto seus efeitos cumulativos. Esse modelo defende que o aprendizado não ocorre devido a novas associações, mas ao aprendizado de um conjunto de capacidades construídas de maneira progressiva por meio de processos de diferenciação, recordação e transferência de aprendizagem. As capacidades aprendidas definidas pelo autor são as seguintes: a) Habilidades mais simples: alocadas no mesmo patamar que as capacidades aprendidas denominadas associações ou conexões estímulo-resposta; b) Discriminações (distinção entre objetos ou símbolos): requerem a prévia aprendizagem de tipos de habilidades bem mais simples, particularmente aquelas que incluem mediadores verbais; c) Conceitos (classes de qualidade de objetos, objetos e eventos): a aprendizagem de conceitos depende da disponibilidade de determinadas discriminações aprendidas anteriormente; d) Regras simples: aprendidas quando outras capacidades, os conceitos, foram previamente 
aprendidas; e) Regras complexas: habilidades intelectuais organizadas pelo indivíduo a partir da combinação de regras mais simples.

A maior contribuição desse modelo, segundo Gagné (1968b), é o conceito de transferência de aprendizagem. Esse conceito afirma que qualquer capacidade aprendida em qualquer estágio da sequência de aprendizagem pode ser aplicada em outro tipo de aprendizagem (situação) diferente da aprendida. Esse tipo de generalização é um processo de transferência interno do aprendiz e pode ser considerado um aspecto importante da capacidade intelectual do mesmo.

Gagné (1968b) também desenvolveu a hierarquia de aprendizagem, a qual representa um conjunto de habilidades intelectuais ordenadas de tal forma que produziriam uma transferência positiva de uma capacidade mais simples para uma mais complexa. Gagné (1972) diferencia seis domínios de aprendizagem e propõe a utilização de tais domínios para distinguir áreas de conteúdos que implicam diferentes tratamentos instrucionais e para elaborar diferentes técnicas de avaliação de resultados de aprendizagem. Os domínios são os seguintes: a) Habilidades motoras: capacidades mediadoras e organizadoras de desempenhos motores; b) Informação verbal: transmissão de fatos, princípios e generalizações; c) Habilidades intelectuais: discriminações, conceitos e regras que constituem as habilidades básicas de um currículo elementar e todas as elaborações que ocorrem durante todo o tempo nos mais avançados assuntos; d) Estratégias cognitivas: habilidades organizadas internamente que orientam o comportamento do indivíduo na aprendizagem, memória e pensamento, e implicam no autogerenciamento da aprendizagem; e) Atitudes: estado mental interno adquirido que influencia as escolhas de ações pessoais feitas pelo indivíduo. As atitudes não são aprendidas pela prática, e um dos modos mais eficientes de promover a mudança de atitudes parece ser a abordagem de modelação comportamental humana e reforçamento comportamental; f) Enterprise: atividade que depende de combinações de informações verbais, habilidades intelectuais e estratégias cognitivas para ocorrer, sendo que todas estão envolvidas com um objetivo comum.

As razões para tal classificação incluem a necessidade de avaliação adequada dos desempenhos esperados em uma instrução e a definição de diferentes tipos de eventos para diferentes tipos de domínios, visando à eficácia do evento. Rodrigues Jr. (2006) destaca que a hierarquia de resultados de aprendizagem proposta por Gagné integra num mesmo continuum a cognição, a psicomotricidade e as atitudes, ao invés de tratá-los de forma separada.

Segundo Gagné (1988), a instrução é um conjunto de eventos externos que podem apoiar e facilitar os processos de aprendizagem, os quais são organizados em sequência, a saber: a) Criar expectativas de sucesso; b) Informar os objetivos ao aprendiz; c) Estimular a lembrança de pré-requisitos; d) Apresentar estímulo; e) Fornecer orientação de aprendizagem; f) Provocar desempenho; g) Fornecer feedback informativo; h) Avaliar o desempenho e i) Aumentar a retenção e a transferência. Nem sempre os eventos instrucionais precisam ser seguidos nesta ordem. Entretanto, todos eles devem ser levados em consideração durante o planejamento de um evento instrucional.

No trabalho de Smith e Ragan (2000) é enfatizado que Gagné descreveu a natureza de uma teoria instrucional na tentativa de relacionar eventos da instrução com resultados de aprendizagem. Segundo os autores, as pesquisas de Gagné em ambientes militares o levaram a questionar alguns aspectos no processo de aprendizagem, o que o fez desenvolver quatro grandes proposições que constituem sua teoria instrucional: a) Os objetivos de aprendizagem podem ser categorizados como resultados de aprendizagem; b) Os resultados de aprendizagem podem ser representados em hierarquias de aprendizagem; c) A aquisição de diferentes resultados requer diferentes condições internas de aprendizagem (grau de prontidão do indivíduo para aprender); d) A aquisição de diferentes resultados requer diferentes eventos de instrução e condições externas de aprendizagem. 
Segundo Abbad e Borges-Andrade (2004), as condições externas, no contexto de programas formais de TD\&E, tratam da definição dos modos de entrega da instrução (cursos presenciais, semipresenciais, à distância, autoinstrucionais), dos meios de ensino (materiais impressos, cd-roms, vídeo conferências, televisão, intranet ou internet), das estratégias de ensino (exposição oral, estudos de caso, exposição dialogada, simulação), das sequências de conteúdos adequadas aos objetivos de ensino que respeitem os princípios de aprendizagem inerentes a cada domínio e da definição dos critérios e medidas de avaliação do alcance dos objetivos propostos inicialmente (testes de papel e lápis, exercícios práticos, relatórios, projetos). Os referidos autores alertam que, além de planejar ações formais de TD\&E de modo sistemático, os planejadores instrucionais devem respeitar também certas condições internas ao indivíduo, necessárias à aprendizagem, como idade, motivação, autoeficácia, locus de controle, inteligência, gênero, idade, história pessoal e profissional, entre outras.

Segundo Richey (2000), o planejamento instrucional tende a ressaltar características dos aprendizes que se relacionam com os assuntos da aula, incluindo pré-requisitos de habilidades, experiências anteriores relacionadas ao tópico da aula ou competências dos aprendizes naquelas estratégias cognitivas requeridas para o domínio do conteúdo. Atualmente, os planejadores estão considerando outras características, tais como atitudes, estilos e estratégias de aprendizagem e experiências culturais trazidas com o aprendiz (Zerbini \& Abbad, 2008). A autora ressalta que os contextos pré e pós-instrucionais também são importantes e devem ser estudados.

$\mathrm{Na}$ análise de Nelson (2000), os resultados das pesquisas de Gagné influenciaram projetos em diversos contextos (treinamento militar, escolas fundamentais, ensino médio e nível superior). Os trabalhos do autor também são relevantes para planejadores da instrução baseada no computador, jogos, simulação e ambientes cooperativos de aprendizagem. Segundo o autor, esforços para racionalizar o processo de planejamento instrucional estão sendo voltados para o desenvolvimento de ferramentas baseadas na gestão do conhecimento que auxiliam planejadores a interpretar problemas e controlar atividades de planejamento. $\mathrm{O}$ autor ressalta que desenvolvimentos recentes fornecidos pelas telecomunicações, tais como a internet, têm aberto oportunidades para atividades educacionais.

Discutidos os processos subjacentes à aprendizagem humana, a seguir são apresentados os conceitos e as características dos eventos formais que induzem a aprendizagem em organizações e ambientes de trabalho, quais sejam: ações de TD\&E, presenciais e a distância, e ações de formação e qualificação profissionais.

\section{$T D \& E$, formação e qualificação profissional: conceitos e características}

Segundo Vargas e Abbad (2006), a pluralidade de conceitos e definições em treinamento e desenvolvimento de pessoal, bem como em diversas outras áreas do conhecimento, pode ser considerada fruto da evolução de pesquisas científicas da área. Entretanto, torna-se necessária a manutenção de tal evolução visando ao avanço do conhecimento na área. Abbad e Borges-Andrade (2004) definem processos de treinamento e desenvolvimento como ações organizacionais que utilizam uma tecnologia instrucional objetivando a aquisição de CHAs para suprir lacunas de desempenho no trabalho e preparar empregados para novas funções. Segundo os autores, essa definição está relacionada à teoria cognitivista (S-O-R), pois sugere que ações organizacionais (S) promovem a aquisição de CHAs (O) que produzem mudanças de comportamento (R) durante o processo de aprendizagem ou no trabalho. 
A expressão treinamento, desenvolvimento e educação (TD\&E) surgiu da expressão criada por Nadler (1984) desenvolvimento de recursos humanos, a qual, segundo Vargas e Abbad (2006), contempla originalmente os três grandes conceitos: treinamento, desenvolvimento e educação. Entretanto, a expressão não se consolidou na forma original proposta pelo autor no contexto nacional e internacional, pois os profissionais da área de treinamento passaram a usála sem levar em consideração a intenção original composta pelos três conceitos. A expressão desenvolvimento de recursos humanos passou a ser usada por diferentes pessoas e com diferentes significados.

Segundo Vargas e Abbad (2006), outra razão para que a expressão treinamento e desenvolvimento prevalecesse sobre desenvolvimento de recursos humanos consiste no fato de que programas educacionais nas organizações, durante muito tempo, eram dirigidos apenas aos níveis gerenciais e à alta administração, ao passo que, para a maioria dos empregados, eram ofertadas ações de treinamentos pontuais para as suas funções. Para as referidas autoras, com o surgimento das universidades corporativas a partir dos anos de 1990, o conceito de educação ficou em evidência dentro das organizações. Era preciso acompanhar as mudanças no mundo do trabalho e oferecer programas educacionais a todos os empregados na organização, bem como a toda a cadeia de valor, incluindo terceirizados, fornecedores, clientes, entre outros. Portanto, nos dias atuais, é necessário agregar o conceito de educação aos conceitos de treinamento e desenvolvimento.

A diferença crítica entre os conceitos de TD\&E, refere-se ao fato de que programas de desenvolvimento são planejados e direcionados para o crescimento pessoal do empregado, sem manter relações estritas com trabalho específico. Educação refere-se às oportunidades dadas pela organização ao indivíduo, visando prepará-lo para ocupar cargos diferentes em um outro momento dentro da mesma organização e o treinamento prepara o indivíduo para melhorar o desempenho no cargo atual (Abbad \& Borges-Andrade, 2004; Nadler, 1984; Sallorenzo, 2000).

Os conceitos de informação e instrução também podem ser considerados formas de indução de aprendizagem. Segundo Rosemberg (2002), no contexto de treinamentos oferecidos a distância, via internet, é importante diferenciar tais conceitos, já que essa modalidade é útil não somente para transmitir uma instrução, como também para disseminar informações, as quais podem servir de apoio aos treinamentos. Segundo o autor, a instrução objetiva o alcance de um resultado de aprendizado específico, mediante planejamento sistemático realizado pela equipe de planejadores instrucionais. A informação consiste em uma organização específica do conteúdo e os objetivos são definidos pelos próprios usuários que buscam a apresentação e a transmissão eficaz de conteúdos.

Para Vargas e Abbad (2006), informação pode ser definida como módulos ou unidades de conteúdos disponibilizados, principalmente, por meio das novas tecnologias da informação e comunicação, tais como portais corporativos, links e bibliotecas virtuais. Instrução, segundo as autoras, define-se como uma estruturação mais simples de ações instrucionais. Em resumo: informação refere-se aos processos de gerenciamento de conhecimentos e instrução relacionase à formulação sistemática de objetivos específicos e definição de métodos de ensino. A Figura 2 representa a relação entre os conceitos de TD\&E proposta por Carvalho (2003) e Zerbini (2003). 
Figura 2: Relação entre os conceitos de TD\&E

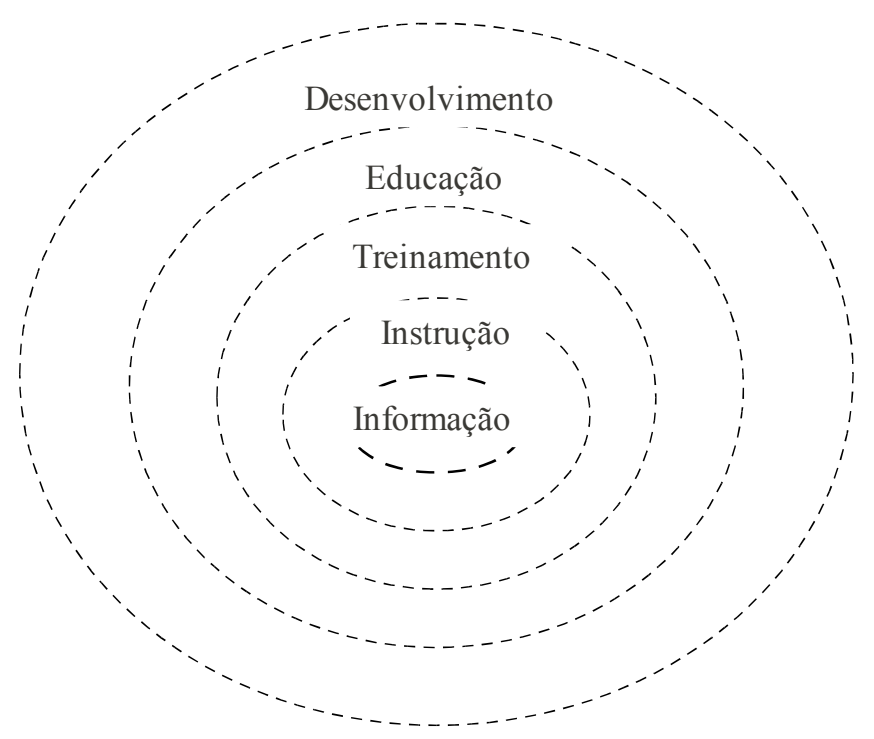

Fonte: Carvalho (2003) e Zerbini (2003).

A razão da utilização de linhas tracejadas justifica-se pelo enfraquecimento das fronteiras entre os conceitos das diferentes ações organizacionais existentes. Abbad e BorgesAndrade (2004) alertam para o fato de que, atualmente, as organizações e os indivíduos participam de treinamentos visando melhorar o desempenho, não somente no cargo atual, como também em cargos futuros e em outras organizações. Segundo Zerbini e Abbad (2005), as ações de desenvolvimento, educação, treinamento, instrução e informação, quando usadas adequadamente e em conjunto, visam promover e acelerar o processo de aprendizagem nas organizações. Nesse sentido, Zerbini e Abbad (2005) definem treinamento como um conjunto de ações educacionais, sistematicamente planejadas, que facilitam o aperfeiçoamento e a aquisição de conhecimentos, habilidades e atitudes (CHAs), podendo ser aplicados no trabalho atual ou futuro, em empresas diferentes e, no caso de participantes autônomos, aplicados nas diversas atividades profissionais desenvolvidas pelo indivíduo.

Quanto ao referencial teórico utilizado na maioria das pesquisas nacionais e estrangeiras sobre ações de TD\&E, a abordagem mais utilizada é a abordagem sistêmica (Borges-Andrade \& Abbad, 1996). Os elementos que compõem o sistema de treinamento são: avaliação de necessidades de treinamento, planejamento e execução de treinamento e avaliação de treinamento, sendo que esses mantêm entre si trocas constantes de informações e resultados.

O subsistema avaliação de necessidades de treinamento identifica as competências necessárias que uma organização precisa desenvolver para alcançar seus objetivos, os CHAs que um indivíduo deve apresentar para desempenhar sua função, bem como, identifica os indivíduos que necessitam de treinamento por não apresentarem esses CHAs desenvolvidos. Em seguida, a ação educacional é planejada e executada a partir da definição dos objetivos instrucionais, definição do conteúdo, da sequência do ensino e da escolha dos meios e estratégias instrucionais mais adequados para se alcançar os objetivos descritos. Por fim, a avaliação de treinamento fornece ao sistema informações sistemáticas sobre lacunas no desempenho dos indivíduos e dos instrutores, identifica falhas no planejamento de procedimentos instrucionais, indica se o treinamento foi positivo para os indivíduos e 
organizações em termos de aplicabilidade e utilidade, informa o quanto as habilidades aprendidas estão sendo aplicadas no trabalho ou na vida profissional dos indivíduos e quais aspectos facilitam ou dificultam este processo (Borges-Andrade \& Abbad, 1996).

Em análise da literatura nacional e estrangeira, Abbad, Carvalho e Zerbini (2006) observam que, de modo geral, os sistemas de treinamento em ambientes organizacionais não utilizam avaliações sistemáticas de necessidades de treinamento, não as relacionam às competências organizacionais ou as alinham às estratégias organizacionais, fazem pequeno esforço de avaliação de aprendizagem e investem pouco em planejamento instrucional de cursos presenciais. Além disso, as autoras destacam a utilização frequente de treinamentos presenciais que privilegiam poucas pessoas da organização, em detrimento de treinamentos a distância, que poderiam alcançar um número bem maior de participantes de diferentes unidades da organização. Observa-se também a ausência de avaliações sistemáticas de treinamento em diferentes níveis e pequena produção tecnológica em avaliação de treinamentos baseados na web (TBWs) e treinamentos baseado em computador (TBCs).

Segundo as autoras supracitadas, a ampliação do acesso a treinamento, formação e qualificação profissionais vem sendo viabilizada pela aplicação das novas tecnologias de informação e comunicação em projetos de educação a distância. Os TBCs e os TBWs, somados às tecnologias tradicionais de disponibilização de conteúdos como o material impresso, materiais em vídeo e áudio, correspondência, vêm se tornando ferramentas fundamentais para capacitação e qualificação profissional dos trabalhadores em ambientes diversificados de trabalho.

Segundo Vargas e Abbad (2006), existem diversas definições para descrever o processo de ensino-aprendizagem que ocorre a distância. Belloni (1999) relata que as definições em EaD são elaboradas pela descrição de suas características, em função de comparações entre as modalidades presencial e a distância. Vargas (2003) acrescenta que, além das definições serem muito explicativas sobre as características que envolvem o processo de ensino-aprendizagem a distância, a literatura em educação a distância apresenta uma confusão conceitual preocupante. A autora, então, propôs uma definição clara e objetiva do que considera educação a distância: "uma modalidade de ensino/aprendizagem que rompe as barreiras do tempo e do espaço, promovendo diferentes formas de interação entre alunos e professores" (p. 292).

Quanto ao termo treinamento a distância (TaD), Vargas (2004) o associa ao contexto específico do ambiente organizacional. Porém, em função do enfraquecimento de fronteiras entre os conceitos de TD\&E, já discutido anteriormente, e das características relacionadas aos processos de educação a distância e aprendizagem aberta, Zerbini e Abbad (2005) definem treinamento a distância como um conjunto de ações educacionais sistematicamente planejadas, desenvolvidas na maior parte do tempo no contexto de flexibilidade espacial e temporal entre professor e aluno, de sincronidade e assincronidade, interação e interatividade (podendo incluir personalização), e de abertura dos espaços físicos, utilizando-se de meios tecnológicos, tais como, material impresso, CD Rom, Internet, Intranet, fitas de vídeo, videoconferências, entre outros, que facilitam o aperfeiçoamento e a aquisição de CHAs através do autogerenciamento da aprendizagem do indivíduo. A definição de treinamento de Zerbini e Abbad (2005) assume que os CHAs adquiridos podem ser aplicados no trabalho atual ou futuro, em empresas diferentes, e, no caso de usuários autônomos em programas de formação e qualificação profissional, aplicados nas diversas atividades profissionais desenvolvidas pelo indivíduo.

Bolzam (1998) relata que muitas empresas usam $\mathrm{TaD}$ para capacitar seus funcionários e economizam em gastos com hospedagem e transporte, típicos de treinamentos presenciais. Rosenberg (2002) ressalta vantagens da utilização de TBWs, entre elas: o alcance de um grande número de pessoas ao mesmo tempo quando as práticas e recursos de uma empresa precisam mudar rapidamente; a atualização instantânea dos conteúdos a serem transmitidos

\section{8}


para os funcionários; a criação de comunidades de aprendizagem, nas quais compartilham-se conhecimentos e experiências mesmo após o término dos treinamentos.

$\mathrm{O} \mathrm{TaD}$, além das vantagens apresentadas, pode facilitar o desenvolvimento de carreiras dentro e fora das organizações por meio de currículos e trilhas educacionais voltados à educação continuada em Universidades Corporativas (UCs). Meister (1999) afirma que uma universidade corporativa é uma unidade educativa dentro das organizações, cuja missão é garantir o aprendizado contínuo de toda a cadeia de valor, incluindo empregados, terceirizados, fornecedores, clientes etc. Assim sendo, passam a constituir o público-alvo das UCs toda a organização e também os atores que com ela estabelecem algum tipo de relação. Portanto, torna-se necessário, nos dias atuais, a implantação de uma nova sistemática de educação corporativa que extrapola consideravelmente os tradicionais limites definidos pelos centros de TD\&E.

Educação corporativa e universidade corporativa, segundo Vargas e Abbad (2006), são conceitos similares, porém, universidade corporativa é um termo mais restritivo, já que nem toda organização tem condições ou precisa implementar essa ferramenta de gestão em sua estrutura. $\mathrm{O}$ conceito de educação corporativa pode ser entendido como programa educacional que envolve os interesses dos indivíduos, equipes e organização, diferente do conjunto de treinamentos tradicionais que visavam apenas ao desenvolvimento de competências específicas dos indivíduos. Para Zerbini, Brauer, Meneses e Abbad (2006), sistemáticas como as pretendidas pelas UCs passam a se articular substancialmente com projetos da gestão da informação e do conhecimento, principalmente no que diz respeito às novas tecnologias necessárias à entrega de ações educacionais a públicos-alvos muito mais amplos do que os visados pelos centros tradicionais. Neste sentido, Eboli (2002) afirma que as experiências mais bem-sucedidas de universidades corporativas estão fundamentadas na $\mathrm{EaD}$ e na utilização intensiva da tecnologia, em suas mais diversas formas, favorecendo um ambiente organizacional propício à aprendizagem ativa, contínua e compartilhada.

Para que os indivíduos consigam atender às novas exigências do mercado de trabalho é preciso garantir a aquisição de novas competências continuamente. Programas de formação e qualificação profissional contribuem para que isso ocorra, sem que o indivíduo esteja necessariamente vinculado a uma corporação. Para Mourão e Puente-Palacios (2006), a formação profissional aproxima-se do conceito de treinamento e desenvolvimento, pois não deixa de ser uma estratégia operacional de busca por qualidade e produtividade. Para Bastos (2006), em função de todas estas exigências do mercado de trabalho, é importante promover a discussão sobre a questão da qualificação e formação do trabalhador. Tal discussão, segundo Mourão (2004), vem sendo feita por instituições de ensino superior, escolas técnicas, órgãos de governo, sindicatos e empresas que compõem o sistema produtivo, já que a formação profissional, nos dias atuais, está diretamente ligada a este sistema.

Segundo Cattani (2000), formação profissional consiste em processos educativos ofertados por escolas ou empresas que promovem a aquisição e o desenvolvimento de conhecimentos teóricos, técnicos e operacionais associados à produção de bens e serviços. Para Mourão e Puente-Palacios (2006) há quatro aspectos presentes na definição de Cattani (2000) importantes de ser destacados: a natureza processual da formação profissional; sua relação com o sistema educacional; escolas e empresas são locais onde a formação profissional pode ocorrer; e finalmente, por ter como objetivo principal fornecer conhecimentos teóricos, técnicos e operacionais relacionados ao sistema produtivo. Cattani (2000) ressalta que a maior parte das demandas associadas à formação profissional é originária das empresas e que os processos educativos são incorporados como estratégia operacional na busca da qualidade e da produtividade. Por outro lado, a formação profissional, associada às demandas advindas dos trabalhadores, assume características diferentes, tais como o desenvolvimento da autonomia e de autovalorização pessoal e profissional. 
Segundo Mourão e Puente-Palacios (2006), qualificação profissional define-se como um conjunto de habilidades, conhecimentos e competências de que o indivíduo dispõe para o seu desempenho profissional. Laranjeira (2000), no entanto, alerta que a adoção de uma só definição de qualificação profissional diante de vários contextos e diferentes fases do desenvolvimento industrial no Brasil é questionável. O relatório do Departamento Intersindical de Estatística e Estudos Socioeconômicos, o DIEESE (1998), por exemplo, ao apresentar o histórico da formação e qualificação profissional no Brasil, enfatiza que até muito recentemente tais ações seguiram o modelo fordista, o qual qualifica um número reduzido de profissionais, deixando que a maior parte dos trabalhadores seja treinada no próprio local de trabalho para suas tarefas específicas.

Para Fleury (2002), o parâmetro do conceito de competência decorre das tarefas e das atribuições de um determinado cargo. Aproxima-se assim, do conceito de qualificação profissional ao definir uma série de requisitos necessários ao cumprimento das atribuições atreladas a cada posição na organização. Esta aproximação entre os conceitos de competência e de qualificação profissional, segundo Ruas (2005), decorre principalmente das características que marcavam o mundo corporativo há quase 30 anos, tais como a prevalência do emprego formal, do trabalho predominantemente industrial, das atividades previsíveis e da forte base sindical. No entanto, o autor ressalta que, atualmente, face à aceleração da concorrência, do trabalho informal e sazonal e da baixa previsibilidade dos negócios e serviços, não é mais suficiente que os indivíduos possuam tais conjuntos de competências, mas, sim, que eles sejam capazes de mobilizá-las em resposta a determinada demanda organizacional. Nesse sentido, entre as várias conotações associadas ao conceito em questão, merecem destaque as abordagens que buscam vincular competência e desempenho organizacional, conforme sugerem Boyatzis (1982), Spencer e Spencer (1983), Ruas (2005), entre outros pesquisadores.

Diante das características analisadas, verifica-se que, nos dias atuais, a formação e qualificação profissional têm como principais pressupostos uma educação básica de qualidade, flexibilidade de conteúdos, formação continuada, e elementos comportamentais, tais como iniciativa, participação e envolvimento.

\section{Considerações finais}

Após as discussões realizadas sobre os processos de aprendizagem induzida por meio de ações formais de TD\&E, formação e qualificação profissional, observa-se que, na atualidade, não é suficiente atender apenas aos requisitos formais especificados nos cargos corporativos, mas demonstrar integração e aplicação de suas competências em reposta às variadas situações de trabalho. Nesse sentido, a aprendizagem induzida, por meio de ações instrucionais formais, pode promover o desenvolvimento de competências que atendam às demandas decorrentes de diferentes ambientes de trabalho e oferecer, igualmente a todos os indivíduos, oportunidades de aprimoramento pessoal e profissional contínuo, conforme a descrição dos quatro pilares da Unesco para a educação do século XXI, quais sejam: saber ser (desenvolvimento pessoal), saber fazer (competência produtiva), saber conhecer (competência cognitiva) e saber conviver (desenvolvimento social).

Associado a tais demandas, emerge um dos grandes desafios do Brasil, que é promover um processo de desenvolvimento sustentável em resposta a fenômenos como a globalização, avanços tecnológicos, empregabilidade, livre comércio, aumento das exportações, entre outros. Para atender a essas demandas, os empresários e as forças políticas do país poderiam atuar juntos no sentido de ampliar a oferta de programas de educação e treinamento à população, além de incentivar e promover pesquisa e desenvolvimento. 
Em função disso, as organizações têm utilizado com frequência as modalidades de ensino presenciais e a distância para ofertar programas instrucionais personalizados de acordo com as necessidades organizacionais. É neste momento que a importância de entender os processos subjacentes à aprendizagem humana no trabalho se justifica. Torna-se fundamental que os profissionais que atuam diretamente com ações formais de aprendizagem em contexto de organização e trabalho fundamentem a sua prática profissional em estudos da administração, da educação, e da psicologia instrucional, organizacional e do trabalho.

Por fim, sugere-se que os profissionais interessados no tema consultem, inicialmente, os trabalhos desenvolvidos por Gagné. Richey (2000) acredita (e as autoras do presente artigo concordam!) que as ideias de Gagné para o planejamento instrucional são bases para a maioria dos modelos atuais de planejamento instrucional, e ainda são dominantes entre os planejadores que têm sido formalmente treinados na área. Para a autora, as orientações básicas do trabalho de Gagné continuarão a ter influência na área de planejamento instrucional mesmo com a emergência de novas teorias de aprendizagem.

\section{Referências}

Abbad, G. (1999). Um modelo integrado de avaliação do impacto do treinamento no trabalho - IMPACT. Tese de Doutorado, Instituto de Psicologia, Universidade de Brasília, Brasília.

Abbad, G. \& Borges-Andrade, J. E. (2004). Aprendizagem humana em organizações de Trabalho. In J. C. Zanelli, J. E. Borges-Andrade \& A. V. B. Bastos (Orgs.), Psicologia, Organizações e Trabalho no Brasil (pp. 237-275). Porto Alegre: Artmed.

Abbad, G., Carvalho, R. S. \& Zerbini, T. (2006). Evasão em curso via internet: explorando variáveis explicativas. Revista de Administração de Empresas Eletrônica, 5 (2), 1-26.

Abbad, G., Nogueira, R. \& Walter, A. M. (2006). Abordagens instrucionais em planejamento de TD\&E. In J. E. Borges-Andrade, G. Abbad \& L. Mourão (Orgs.), Treinamento, desenvolvimento e educação em organizações e trabalho: fundamentos para a gestão de pessoas (pp. 255-281). Porto Alegre: Artmed.

Abbad, G., Zerbini, T., Carvalho, R. S. \& Meneses, P. P. M. (2006). Planejamento instrucional em TD\&E. In J. E. Borges-Andrade, G. Abbad \& L. Mourão (Orgs.), Treinamento, desenvolvimento e educação em organizações e trabalho: fundamentos para a gestão de pessoas (pp. 289-321). Porto Alegre: Artmed.

Baldwin, T. T. \& Ford, J. K. (1988). Transfer of training: A review and directions for future research. Personnel Psychology, 41 (1), 63-105.

Bastos, A. V. B. (2006). Trabalho e qualificação: questões conceituais e desafios postos pelo cenário de reestruturação produtiva. In J. E. Borges-Andrade, G. Abbad \& L. Mourão (Orgs.), Treinamento, desenvolvimento e educação em organizações e trabalho: fundamentos para a gestão de pessoas (pp. 23-40). Porto Alegre: Artmed.

Belloni, M. L. (1999). Educação a distância. Campinas: Autores Associados.

Bloom, B., Engelhart, M. D., Furst, E. J., Hill, W. H. \& Krathwohl, D. R. (1972a). Taxonomia dos Objetivos Educacionais - Domínio Afetivo. Porto Alegre:Globo.

Bloom, B., Engelhart, M. D., Furst, E. J., Hill, W. H. \& Krathwohl, D. R. (1972b). Taxonomia dos Objetivos Educacionais - Domínio Cognitivo. Porto Alegre: Globo.

Bolzan, R. de F. F. de A. (1998). O conhecimento tecnológico e o paradigma educacional. Dissertação de Mestrado, Engenharia de Produção, Universidade Federal de Santa Catarina, Florianópolis.

Borges-Andrade, J. E. \& Abbad, G. (1996). Treinamento e desenvolvimento: reflexões sobre suas pesquisas científicas. Revista de Administração, 31 (2), 112-125.

Boyatizis, R. (1982). The competent manager: a model for effective performance. New York: Wiley.

Carvalho, R. S. (2003). Avaliação de treinamento a distância via internet: reação, suporte à transferência e impacto do treinamento no trabalho. Dissertação de Mestrado, Instituto de Psicologia, Universidade de Brasília, Brasília. 
Cattani, A. D. (2000). Formação profissional (verbete). In A. D. Cattani (Org.), Trabalho e tecnologia: dicionário crítico. Petrópolis: Vozes.

Departamento Intersindical de Estatística e Estudos Sócio-Econômicos (1998). Formação profissional: um novo espaço de negociação (Série Pesquisas DIEESE, 14). São Paulo: DIEESE.

Eboli, M. (2002). Desenvolvimento das pessoas e educação corporativa. In M. T. L. Fleury (Org.), As pessoas na organização (pp. 185-216). São Paulo: Gente.

Fleury, M. T. L. (2002). A gestão de competência e a estratégia organizacional. In M. T. L. Fleury (Org.), As pessoas na organização (pp. 51-61). São Paulo: Gente.

Ford, J. K. \& Kraiger, K. (1995). The application of cognitive constructs and principles to the instructional systems design model of training: implications for needs assessment, design, and transfer. International Review of Industrial and Organizational Psychology, 10, 1-48.

Gagné, R. M. (1968a). Contributions of learning to human development. Psychological Review, 75, 177-191.

Gagné, R. M. (1968b). Learning hierarchies. Educational Psychologist, 6, 1-9.

Gagné, R. M. (1972). Domains of learning. Interchange, 3, 1-8.

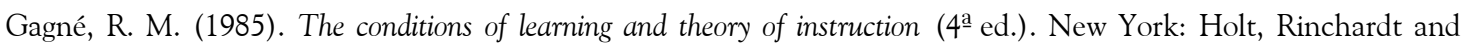
Winston.

Gagné, R. M. (1988). Mastery learning and instructional design. Performance Improvement Quarterly, 1 (1), 7-18.

Hamblin, A. C. (1978). Avaliação e controle do treinamento. São Paulo: McGraw-Hill.

Laranjeira, S. M. G. (2000). Qualificação (verbete). In A. D. Cattani (Org.), Trabalho e tecnologia: dicionário crítico. Petrópolis: Vozes.

Meister, J. C. (1999). A gestão do capital intelectual através das Universidades Corporativas. São Paulo: Markon.

Mourão, L. (2004). Avaliação de programas públicos de treinamento: um estudo sobre o impacto no trabalho e na geração de emprego. Tese de Doutorado, Instituto de Psicologia, Universidade de Brasília, Brasília.

Mourão, L. \& Puente-Palacios, K. E. (2006). Formação Profissional. In J. E. Borges-Andrade, G. Abbad \& L. Mourão (Orgs.), Treinamento, desenvolvimento e educação em organizações e trabalho: fundamentos para a gestão de pessoas (pp. 41-64). Porto Alegre: Artmed.

Nadler, L. (1984). The handbook of human resources development. New York: Wiley.

Nelson, W. A. (2000). Gagné and the new technologies of instruction. In R. C. Richey (Org.), The legacy of Robert M. Gagné. New York: Syracuse University.

Pantoja, M. J. \& Borges-Andrade, J. E. (2002). Uma abordagem multinível para o estudo da aprendizagem e transferência nas organizações. In Anais do XXVI ENANPAD. Salvador: Associação Nacional dos Programas de Pós-Graduação em Administração.

Pilati, R. \& Abbad, G. (2005). Análise fatorial confirmatória da escala de impacto do treinamento no trabalho. Psicologia: Teoria e Pesquisai 21 (1), 43-51.

Richey, R. C. (2000). The legacy of Robert M. Gagné. New York: Syracuse University.

Rodrigues Jr., J.F. (2006). Taxonomia de objetivos em TD\&E. In J. E. Borges-Andrade, G. Abbad \& L. Mourão (Orgs.), Treinamento, desenvolvimento e educação em organizações e trabalho: fundamentos para a gestão de pessoas (pp. 282-288). Porto Alegre: Artmed.

Rosenberg, M. J. (2002). E-learning: estratégias para a transmissão do conhecimento na era digital. São Paulo: Makron.

Ruas, R. (2005). Gestão por competências: uma contribuição à estratégia das organizações. In R. Ruas, C. S. Antonello \& L. H. Boff (Orgs.), Os novos horizontes da gestão: aprendizagem organizacional e competências (pp. 34-55). Porto Alegre: Bookman.

Sallorenzo, L. H. (2000). Avaliação de impacto de treinamento no trabalho: analisando e comparando modelos de predição. Dissertação de Mestrado, Instituto de Psicologia, Universidade de Brasília, Brasília.

Smith, P. L. \& Ragan, T. J. (2000). The Impact of R. M. Gagné's Work on Instructional Theory. In R. C. Richey (Org.), The Legacy of Robert M. Gagné. New York: Syracuse University.

Spencer, L. \& Spencer, S. (1993). Competence at work. New York: John Wiley \& Sons.

Vargas, M. R. M. (2003). Educação a distância no contexto da mudança organizacional. In S. M. V. Lima (Org.), Mudança organizacional: teoria e gestão (pp. 291-315). Rio de Janeiro: FGV. 
Vargas, M. R. M. (2004). Barreiras à implantação de programas de educação e treinamento a distância. Tese de Doutorado, Instituto de Psicologia, Universidade de Brasília, Brasília.

Vargas, M. R. M. \& Abbad, G. (2006). Bases conceituais em treinamento, desenvolvimento e educação - TD\&E. In J. E. Borges-Andrade, G. Abbad \& L. Mourão (Orgs.), Treinamento, desenvolvimento e educação em organizações e trabalho: fundamentos para a gestão de pessoas (pp. 137-158). Porto Alegre: Artmed.

Zerbini, T. (2003). Estratégias de aprendizagem, reações aos procedimentos de um curso via internet, reações ao tutor e impacto do treinamento no trabalho. Dissertação de Mestrado, Instituto de Psicologia, Universidade de Brasília, Brasília.

Zerbini, T. \& Abbad, G. (2005). Impacto de treinamento no trabalho via internet. Revista de Administração de Empresas Eletrônica, 4 (2), 1-21.

Zerbini, T. \& Abbad, G. (2008). Estratégias de aprendizagem em curso a distância: validação de uma escala. PsicoUSF, 13 (2), 177-187.

Zerbini, T., Brauer, S., Meneses, P. P. M. \& Abbad, G. (2006). Percepções sobre educação a aistância: limitações e restrições à implantação da Universidade Corporativa do Banco Central do Brasil. In Anais do XXX ENANPAD. Salvador: Associação Nacional dos Programas de Pós-Graduação em Administração.

\section{Endereço para correspondência}

thais.zerbini@gmail.com,gardenia.abbad@gmail.com

Recebido em: 01/12/2008

Revisado em: 14/09/2009

Aprovado em: 05/02/2010 